\title{
Hyaluronic acid vs. physiological saline for enlarging deficient gingival papillae: a randomized controlled clinical trial and an in vitro study
}

\author{
Jing $\mathrm{Ni}^{1}$, Zhe Zhong ${ }^{2}$, Yifan $\mathrm{Wu}^{1}$, Rong Shu ${ }^{1}$, Yiqun $\mathrm{Wu}^{3}$, Chaolun $\mathrm{Li}^{3}$ \\ ${ }^{1}$ Department of Periodontology, Shanghai Ninth People's Hospital, Shanghai Jiao Tong University School of Medicine, College of Stomatology, \\ Shanghai Jiao Tong University, National Center for Stomatology, National Clinical Research Center for Oral Diseases, Shanghai Key Laboratory \\ of Stomatology, Shanghai, China; ${ }^{2}$ Center for Dental Research, Loma Linda University School of Dentistry, Loma Linda, CA, USA; ${ }^{3} 2$ nd Dental \\ Center, Shanghai Ninth People's Hospital, Shanghai Jiao Tong University School of Medicine, Shanghai Key Laboratory of Stomatology College \\ of Stomatology, Shanghai Jiao Tong University, National Center for Stomatology, National Clinical Research Center for Oral Diseases, Shanghai, \\ China \\ Contributions: (I) Conception and design: C Li, Y Wu, Z Zhong; (II) Administrative support: J Ni, C Li; (III) Provision of study materials or patients: \\ Y Wu, R Shu; (IV) Collection and assembly of data: J Ni; (V) Data analysis and interpretation: J Ni, Z Zhong; (VI) Manuscript writing: All authors; \\ (VII) Final approval of the manuscript: All authors. \\ Correspondence to: Chaolun Li; Yiqun Wu. 2nd Dental Center, Ninth People's Hospital, Shanghai Jiao Tong University School of Medicine, 639 \\ Zhizao Ju Road, Shanghai 200011, China. Email: chaolunli@126.com; yiqunwu@hotmail.com.
}

Background: loss of the interdental papillae leads to the formation of a black triangle, which compromises smile esthetics and contributes to food impaction and plaque accumulation. The aim of this study was to evaluate the efficacy of the injection of hyaluronic acid (HA) and compare it to that of physiological saline solution in the restoration of deficient gingival papillae in vivo and in vitro.

Methods: Twenty-four patients with 68 deficient gingival papillae were recruited for this clinical trial with a split-mouth design. The deficient gingival papillae on one side of the anterior maxilla were injected with $\mathrm{HA}$, and those on the other side were injected with physiological saline solution. The heights of the gingival papillae and the areas of the black triangles were measured from clinical photographs obtained before and 6 and 12 months after treatment. Additionally, the proliferation and migration of gingival fibroblasts were evaluated after HA and physiological saline treatment by an in vitro study.

Results: the results revealed that the injection of HA yielded 0.198 and $0.28 \mathrm{~mm}$ gingival papilla increasement at 6 and 12 months, respectively, relative to the baseline $(\mathrm{P}<0.05)$. However, deficient gingival papillae also grew by $0.278 \mathrm{~mm}$ at 12 months in the group that received physiological saline solution $(\mathrm{P}<0.05)$. The injection of HA significantly improved deficient gingival papillae 6 months earlier than the injection of physiological saline solution. HA also significantly accelerated the proliferation and migration of gingival fibroblasts in vitro.

Conclusions: The present study confirms that the injection of HA could increase the height of gingival papillae for improving gingival papilla defects. However, the effect is not superior to that of physiological saline solution. This trial was registered in the Chinese Clinical Trial Registry (ChiCTR-ONC-17011781) (28/06/2017). http://www.chictr.org.cn/showproj.aspx?proj=19931

Keywords: Hyaluronic acid (HA); gingival papilla; gingival black triangle; physiological saline solution; gingival fibroblast

Submitted Nov 22, 2020. Accepted for publication Mar 05, 2021.

doi: $10.21037 / \mathrm{atm}-20-7599$

View this article at: http://dx.doi.org/10.21037/atm-20-7599 


\section{Introduction}

Interdental papillae, along with the optimal gingival contours, are crucial for ensuring smile esthetics. Gingival papilla defects in the anterior maxillary region lead to the formation of open gingival embrasures and negative spaces known as "black triangles", which are mostly undesirable to patients with high smile lines. There are several techniques, including restorative procedures (1), orthodontic treatments (2), interdisciplinary approaches (3) and a variety of surgical augmentation procedures (4-8), that can be used to reconstruct the missing gingival papillae. The first three options are costly and time consuming, while surgical techniques for augmenting the hard and soft tissues at the sites of the black triangles are invasive and yield unpredictable outcomes.

Recently, the injection of HA showed promising clinical efficacy in the treatment of gingival papilla defects in several studies. Becker et al. reported that small papillary deficiencies could be improved by injecting hyaluronic gel and that the enhancements were sustained for 6 to 25 months (9). Pi et al. also demonstrated in a rat model that the local injection of HA filler was a meaningful minimally invasive procedure for reducing the areas of black triangles (10). Awartani et al. demonstrated that interdental papilla loss could be improved after 6 months by the injection of HA gel in 10 patients with 17 black triangle sites (11). A study by Bertl et al., however, found that injection of HA adjacent to anterior maxillary implant-supported crowns did not result in any clinically obvious volume augmentation in deficient papillae (12). These conflicting findings indicate that validation of the efficacy of HA injection for the treatment of gingival papillary defects still requires higherlevel clinical evidence. Meanwhile, basic research on the effects of HA on gingival connective tissue is necessary to unveil the underlying mechanisms.

HA is a non-sulfated glycosaminoglycan that is present in many body fluids. It maintains extracellular matrix elasticity and tissue hydration. Asparuhova et al. illustrated that hyaluronan (Regedent AG, Zurich, Switzerland) enhances the proliferation and migration of gingival fibroblasts, which are the main cell type in gingival connective tissue (13). The products of HA also increase the expression of genes encoding type III collagen and transforming growth factor- $\beta 3$ (13), which partly explains the underlying mechanism by which HA in resolves gingival papillary defects. Unfortunately, no clinical study has been conducted on these products to confirm their clinical effects.
In our previous study (14), we verified the remarkable effectiveness of hyaluronic acid (HA) gel (Qi Sheng Biological Agent Company Limited, Shanghai, China) injections in restoring the deficient gingival papillae of natural teeth, especially in patients with thick gingival biotypes. Nevertheless, that study had several shortcomings, such as a small sample size and a lack of a control group. In this study, we further evaluated the efficacy of HA gel injections in the improvement of deficient gingival papillae and the reduction of black triangles and compare it to that of the injection of physiological saline solution with a randomized controlled split-mouth design. We also examined the proliferation and migration of gingival fibroblasts after stimulation with the same HA product and physiological saline product in vitro. We present the following article in accordance with the CONSORT reporting checklist (available at http://dx.doi.org/10.21037/ atm-20-7599).

\section{Methods}

\section{Study population and inclusion criteria}

Our study was designed based on the guidelines from the Consolidated Standards of Reporting Trials (CONSORT) statement (http://www.consort-statement.org/) and was registered in the Chinese Clinical Trial Registry (ChiCTRONC-17011781) http://www.chictr.org.cn/showproj. aspx? proj=19931. We confirmed that all methods were performed in accordance with the relevant guidelines and regulations. In this trial, 24 patients with 68 gingival papilla defects were recruited.

The inclusion criteria were as follows: (I) adults (2070 years old); (II) no systemic disease such as hypertension, coronary artery disease, stroke, or Type II diabetes that would affect periodontal treatment; (III) no fixed prostheses or caries on the studied teeth; (IV) good oral hygiene (fullmouth plaque score <20\%) (15); (V) two or four symmetrical gingival papilla defects (Class I, in which the tip of the interdental papilla lies between the interdental contact point and the most coronal extent of the interproximal cementoenamel junction (CEJ), or Class II, in which the tip of the interdental papilla lies at or apical to the interproximal CEJ but coronal to the apical extent of the facial CEJ (16) in the anterior maxilla; (VI) healthy periodontal tissue or wellcontrolled inflammation, which is defined as $<10 \%$ bleeding sites with probing depths $\leq 3 \mathrm{~mm}$ (17); (VII) no history of periodontal surgery in the last 6 months; (VIII) no smoking; 


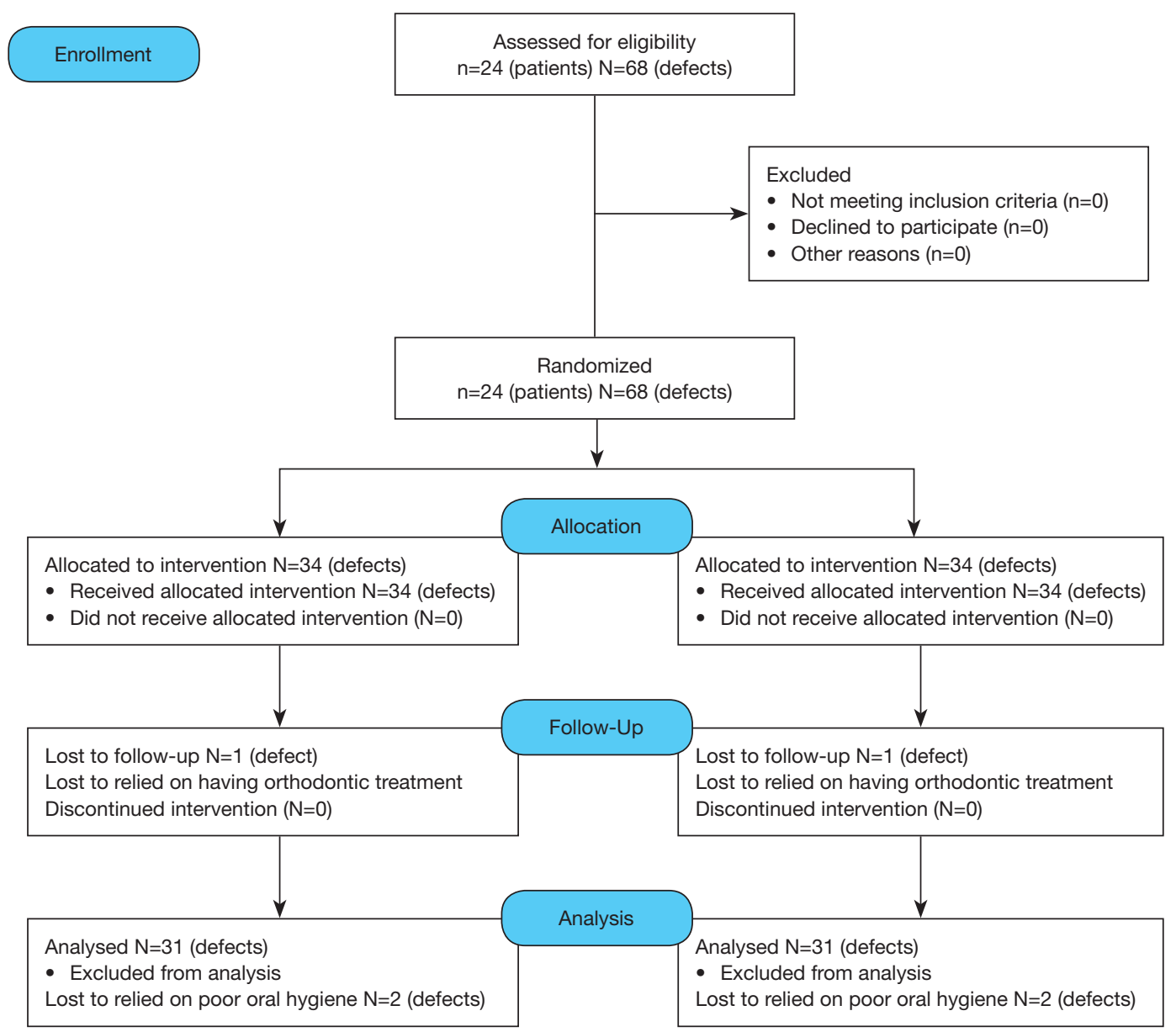

Figure 1 Flow chart of the clinical trial.

(IX) no history of regular medication intake such as calcium channel blockers or cyclosporin A that could affect gingiva metabolism (18).

The study was conducted in accordance with the Declaration of Helsinki (as revised in 2013). The study was approved by the Shanghai Ninth People's Hospital Human Studies Ethics Committee (No. 2016-229-T178). All participants were informed of the objective and risks of the study. Candidates were required to provide signed consent to participate.

\section{Study design}

The study used a split-mouth design, as shown in Figure 1 . All candidates received initial periodontal therapy, which involved the use of scaling, root debridement and the provision of oral hygiene instructions. After one month, the candidates were reevaluated, and those who fulfilled the inclusion criteria were recruited into the study. Blinding was implemented for the trial participants, the image assessors, who made the measurements on the images, and the data collections. An independent researcher created random allocation cards with Microsoft Excel. The random assignment of an odd number indicated that the left side would be used for the test group (HA group) and the right side for the control group (physiological saline solution group). The random assignment of an even number indicated that the right side would be used for the test group (HA group) and the left side for the control group (physiological saline solution group). Yifan Wu performed the follow-up examinations 6 and 12 months after the injections.

\section{Standardized photography and measurement}

Prior to the injections, a standardized clinical photograph of 


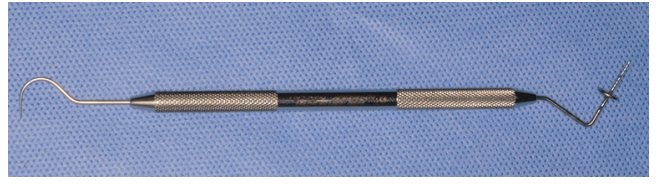

Figure 2 The intraoral scale calibration custom probe with a stop.

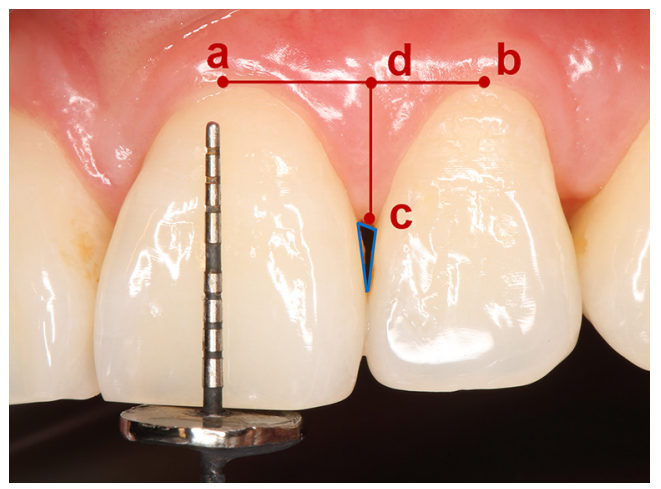

Figure 3 Description of the methodology used to measure the papilla height and papilla triangle areas. Line ab represents the line connecting the highest point of the adjacent teeth's crowns with the gingival papilla defect. Point $\mathrm{c}$ represents the top of the deficient gingival papilla. Line cd is vertical to line ab. cd represents the papilla height, and the papilla triangle area is marked with a blue line.

the gingival papilla defects was taken with a Canon EOS5D Mark III digital camera. The lens of the camera was a Canon EF $100 \mathrm{~m} f / 2.8 \mathrm{~L}$ IS USM, and the camera parameters were ISO $200, \mathrm{f} / 32$ and 1/160s.A 1:1 magnification ratio and $25 \mathrm{~cm}$ fixed distance were maintained during the photo shoot. Meanwhile, one assistant checked that the camera was perpendicular to the long axis of the adjacent teeth and parallel to both the participant's Frankfurt horizontal plane and the ground. An intraoral scale calibration custom probe with a stop was used in the study (Figure 2). The stop could ensure the probe is parallel to the long axis of the tooth.

Afterwards, the height of each gingival papilla (primary outcome measure) and the area of each black triangle (secondary outcome measure) was measured on the clinical image with Digimizer version 4.2 software (Med Calc Software, Belgium), as described in previous study (14). A summary description of the methodology used to measure the papilla height and triangle areas is illustrated in Figure 3. Training for measurement from the clinical images was performed by measuring the papilla height and papilla triangle areas on 10 clinical images that were not associated with the clinical trial.

\section{Injection procedure}

All injection procedures were performed under anesthesia by the same experienced periodontist. The control sites, located on one side of the mouth, were injected with physiological saline solution at the bases of the deficient papillae. The test sites, located on the other side of the mouth, were injected with $16 \mathrm{mg} / \mathrm{mL}$ HA gel (Qi sheng Biological Agent Company Limited, Shanghai, China) at the bases of the deficient papillae. Each site received 0.05 $0.1 \mathrm{~mL}$ of solution. The injection procedures were repeated 3 and 6 weeks after the initial injections.

\section{In vitro study}

\section{Cell culture and identification}

Human gingival (HGF) fibroblasts were obtained from 3 systemically and periodontally healthy patients $(<10 \%$ bleeding sites and probing depths $\leq 3 \mathrm{~mm}$ ) who had undergone crown lengthening. In advance of the procedure, the patients signed informed consent forms. The in vitro study was approved by Shanghai Ninth People's Hospital Human Studies Ethics Committee. The gingival tissue obtained was minced into $1 \mathrm{~mm}$ pieces and cultivated in a $21 \mathrm{~cm}^{2}$ culture dish in DMEM, Dulbecco's modified Eagle's medium (HyClone, USA) supplemented with 10\% fetal calf serum (GIBCO, USA). Cells between passages 4-7 were used in the study. Gingival fibroblasts were identified with morphological observation and immunochemistry.

\section{Cell proliferation assay}

The proliferation rate of HGF cells was determined by CCK-8 (Dojindo, Shanghai, China). HGF cells were inoculated in 96 well plates at a density of $2 \times 10^{3} / 100 \mu \mathrm{L}$ per well. After $24 \mathrm{~h}$ of incubation, $0 \% \mathrm{HA}, 1 \% \mathrm{HA}, 3 \%$ $\mathrm{HA}$ and $6 \% \mathrm{HA}$ were added to the 96 -well plates. The cells were incubated for 1 day, 2 days, 3 days and 4 days, respectively. After CCK-8 was added to the wells, the plate was placed into an incubator for $2 \mathrm{~h}$, and the absorbance value was measured at $450 \mathrm{~nm}$ with a Microplate Reader. Three independent experiments were performed with the cells obtained from the three different patients.

\section{Cell migration assay}

After serum-free starvation for $24 \mathrm{~h}$, the cells were resuspended in DMEM containing 1\% FBS, and the cell 
concentration was adjusted to $1 \times 10^{5}$ cells $/ \mathrm{mL}$. A $150 \mu \mathrm{L}$ cell suspension was inoculated in the upper chamber (Corning, New York, USA). A total of $700 \mu \mathrm{L}$ of $0 \%, 1 \%$ and $13 \%$ $\mathrm{HA}$, which were all diluted in DMEM with $1 \% \mathrm{FBS}$, was added to the lower chamber. After $12 \mathrm{~h}$ of cultivation, the non-migrated cells on the upper chamber surface were wiped with cotton swabs. After 30 minutes of methanol fixation, $0.1 \%$ crystal violet staining was performed. The mean values of 3 visual fields were calculated. Three independent experiments were performed with the cells obtained from the three different patients.

\section{Data analysis}

Data analysis was performed with SPSS (Version 23.0, IBM, Armonk, NY, USA). Differences were considered significant when their $\mathrm{P}$ values were less than 0.05 . Descriptive statistics were calculated and are presented as the mean \pm SD. For the clinical study, the gingival papilla heights and the black triangle areas were compared with repeated-measures analysis of variance (ANOVA). In detail, first, the studentized residuals were generated and analyzed. The results indicated that the data from each group had no outliers (no value beyond \pm 3 ) and followed a normal distribution (all $\mathrm{P}>0.05$ by the Shapiro-Wilk test). If $\mathrm{P}_{\text {Mauchly's test of sphericity }} \geq 0.05$, the $\mathrm{P}$ values of sphericity assumed were adopted; if $\mathrm{P}_{\text {Mauchly's test of sphericity }}<0.05$, and epsilon $(\varepsilon)$ $<0.75$, the $\mathrm{P}$ values of Greenhouse-Geisser were adopted; if $\mathrm{P}_{\text {Mauchly's test of sphericity }}<0.05$ and epsilon $(\varepsilon)>0.75$, the $\mathrm{P}$ values of Huynh-Feldt were adopted. Bonferroni correction was applied to the multiple comparisons. One-way analysis of variance (ANOVA) was adopted in the in vitro study. $\mathrm{P}$ values $<0.05$ were considered significant.

\section{Results}

Twenty-four patients with a total of 68 gingival papilla defects were included in the study. Thirty-four gingival papilla defects were in the test group $(\mathrm{N}=34)$ and treated with HA gel, and 34 gingival papilla defects were in the control group $(\mathrm{N}=34)$ and treated with physiological saline solution. One patient discontinued participation in orthodontic treatment. Two patients' data were removed from the analysis because of poor oral hygiene, which led to gingival swelling. The study ended with completion of the 12-month follow-up and the statistical analysis of the data of the remaining 21 patients with 62 gingival papilla defects. The patients' ages ranged from 28 to 63 years (average
$41.3 \pm 7.73$ years), and $2 / 21$ were males (Table 1 ).

As shown in Table 2, the average increases in gingival papilla height were $0.198 \pm 0.34$ and $0.280 \pm 0.38 \mathrm{~mm}$ at 6 and 12 months respectively, in the test group. In the control group, the average increases in gingival papilla height were $0.135 \pm 0.39$ and $0.278 \pm 0.45 \mathrm{~mm}$ at 6 and 12 months respectively. The average decreases in the black triangle area were $0.26 \pm 0.42$ and $0.45 \pm 0.54 \mathrm{~mm}^{2}$ at 6 and 12 months respectively, in the test group. The average decreases in black triangle area were $0.15 \pm 0.37$ at 6 months and $0.32 \pm 0.50 \mathrm{~mm}^{2}$ at 12 months in the control group. The intragroup comparisons for the test group indicated statistically higher gingival papilla heights and statistically smaller black triangle areas between 6 months and baseline (Table 2, $\mathrm{P}=0.011$ for the gingival papilla height, $\mathrm{P}=0.007$ for the black triangle area). Similar results were shown between 12 months and baseline (Table 2, $\mathrm{P}=0.001$ for the gingival papilla height, $\mathrm{P}<0.001$ for the black triangle area). The intragroup comparisons for the control group indicated no significant difference in either the gingival papilla height or the black triangle area between 6 months and baseline (Table 2, $\mathrm{P}=0.199$ for the gingival papilla height, $\mathrm{P}=0.098$ for the black triangle area). However, significantly different results were observed between baseline and 12 months (Table 2, $\mathrm{P}=0.006$ for the gingival papilla height, $\mathrm{P}=0.004$ for the black triangle area). Intergroup analysis revealed that there was no significant main effect for the changes in gingival papilla heights or the area of black triangles between the test group and the control group (Table 2, $\mathrm{P}=0.078$ for gingival papilla height, $\mathrm{P}=0.826$ for black triangle area). Clinical photographs of representative patients are shown in Figure 4.

HGFs were cultivated with the explant culture method, and fibroblast-like cells could be observed around the tissue edge after 3 days. The cells were passaged after reaching 80-90\% confluence. The obtained cells were spindle shaped under a microscope (Figure 5). The immunochemistry results indicated that the cells were positively stained with vimentin antibody and negatively stained with cytokeratin antibody (Figure 5). The cultured cells were confirmed to be derived from the mesoderm and were not mixed with epithelial cells.

The CCK- 8 results showed that gingival fibroblasts proliferated significantly after stimulation with $1 \% \mathrm{HA}, 3 \%$ $\mathrm{HA}$ and $6 \% \mathrm{HA}$ for 3 days and 4 days relative to the control group (Figure 6). Cell migration assays confirmed that gingival fibroblasts migrated significantly in the $13 \% \mathrm{HA}$ group relative to the $1 \% \mathrm{FBS}$ control group and 1\% HA 
Table 1 Characteristics of the study population

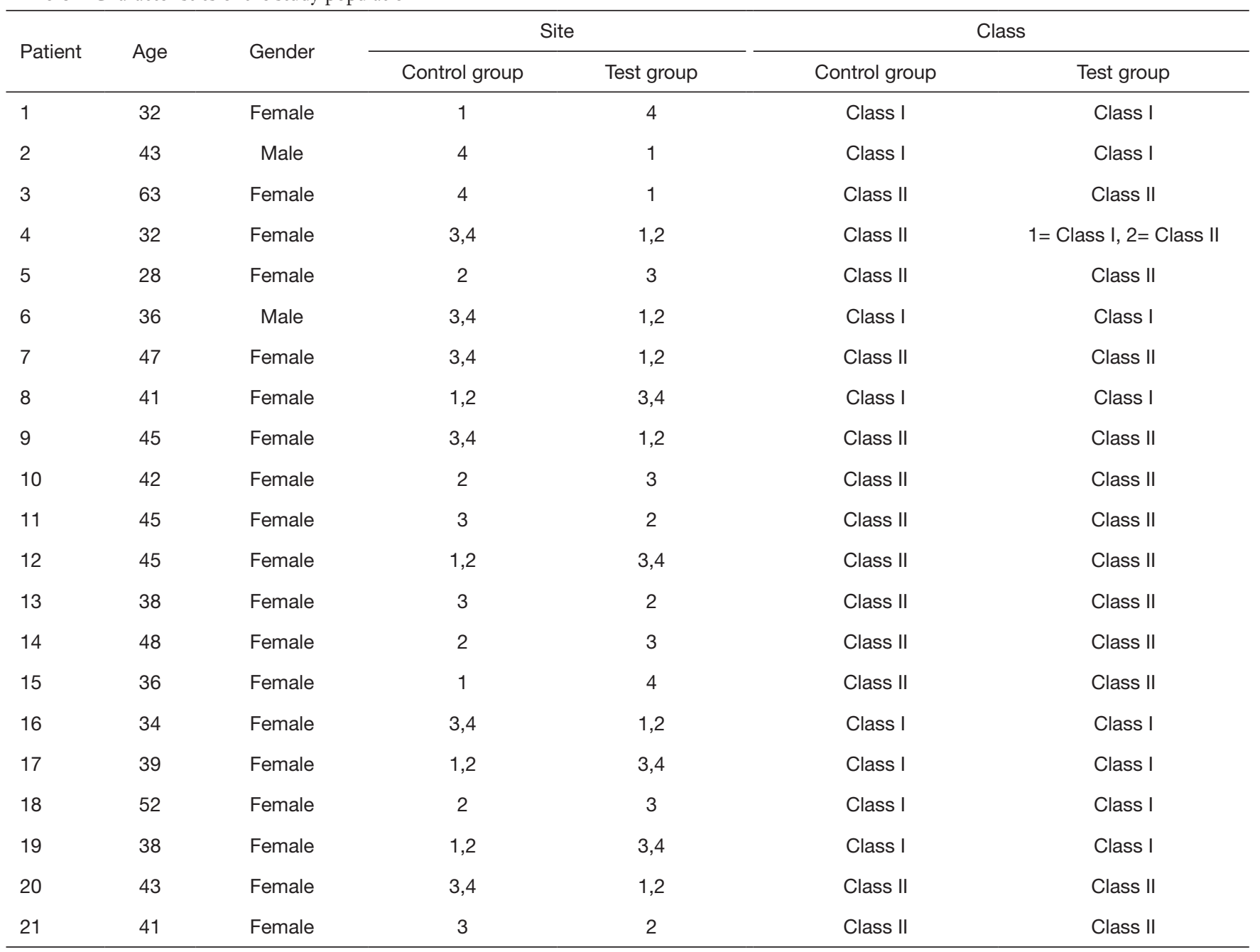

Site 1= interdental papilla between maxillary right canine and maxillary right lateral incisor; Site 2 = interdental papilla between maxillary right lateral incisor and maxillary right central incisor; Site $3=$ interdental papilla between maxillary left lateral incisor and maxillary left central incisor; Site 4 = interdental papilla between maxillary left lateral incisor and maxillary left canine.

Table 2 Clinical data for gingival papilla treated in control and test group at baseline and after 6 and 12 months

\begin{tabular}{|c|c|c|c|c|c|c|c|c|}
\hline Parameter/treatment & Baseline & 6 months & $\Delta 6$ months-BL & $P$ value ${ }^{a}$ & 12 months & $\Delta 12$ months-BL & $P$ value ${ }^{b}$ & $P$ value ${ }^{c}$ \\
\hline Control group ( $\mathrm{N}=31$ ) & $2.99 \pm 1.42$ & $3.12 \pm 1.36$ & $0.135 \pm 0.39$ & 0.199 & $3.26 \pm 1.39$ & $0.278 \pm 0.45$ & $0.006^{*}$ & \\
\hline Test group $(\mathrm{N}=31)$ & $3.25 \pm 1.30$ & $3.45 \pm 1.27$ & $0.198 \pm 0.34$ & $0.011^{*}$ & $3.53 \pm 1.25$ & $0.280 \pm 0.38$ & $0.001^{*}$ & \\
\hline The area of black triangle $\left(\mathrm{mm}^{2}\right)$ & & & & & & & & 0.826 \\
\hline Test group $(\mathrm{N}=31)$ & $1.90 \pm 1.37$ & $1.65 \pm 1.32$ & $-0.260 \pm 0.42$ & $0.007^{\star}$ & $1.45 \pm 1.16$ & $-0.450 \pm 0.54$ & $<0.001^{*}$ & \\
\hline
\end{tabular}

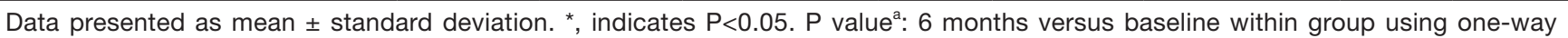
repeated ANOVA corrected by Bonferroni; $P$ value ${ }^{b}: 12$ months versus baseline within group using one-way repeated ANOVA corrected by Bonferroni; $P$ value ${ }^{c}$ : test group versus control group using two-way repeated ANOVA. 

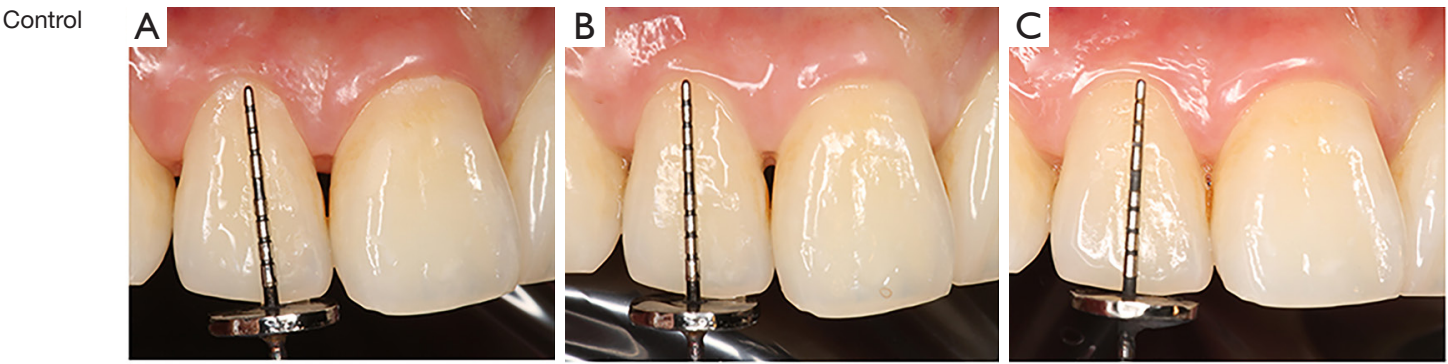

test
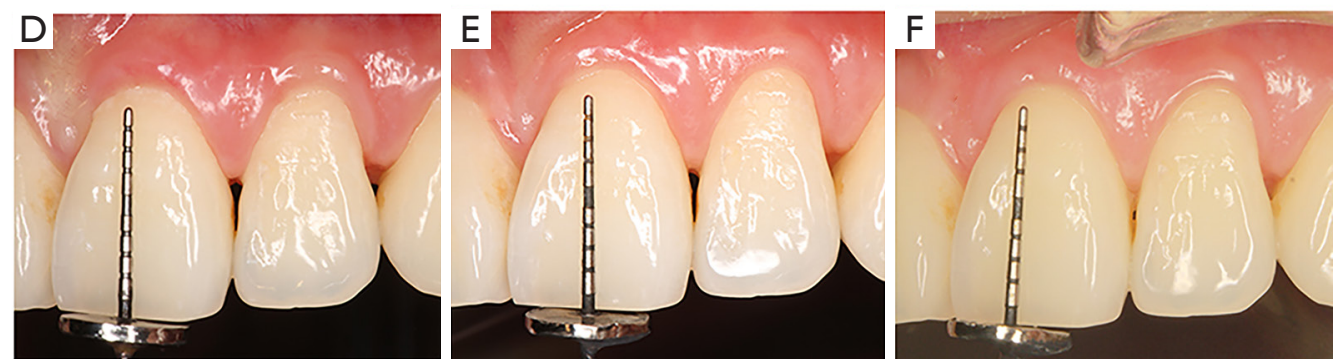

Figure 4 Clinical photographs of representative patients treated with injections of physiological saline solution (control group) and hyaluronic acid gel (test group). A deficient gingival papilla between the right maxillary incisor and the right maxillary lateral incisor is shown after treatment with a physiological saline solution injection at baseline (A) and after 6 months (B) and 12 months (C). A deficient gingival papilla between the left maxillary incisor and the left maxillary lateral incisor is shown after treatment with a hyaluronic acid gel injection at baseline (D) and after 6 months (E) and 12 months (F).
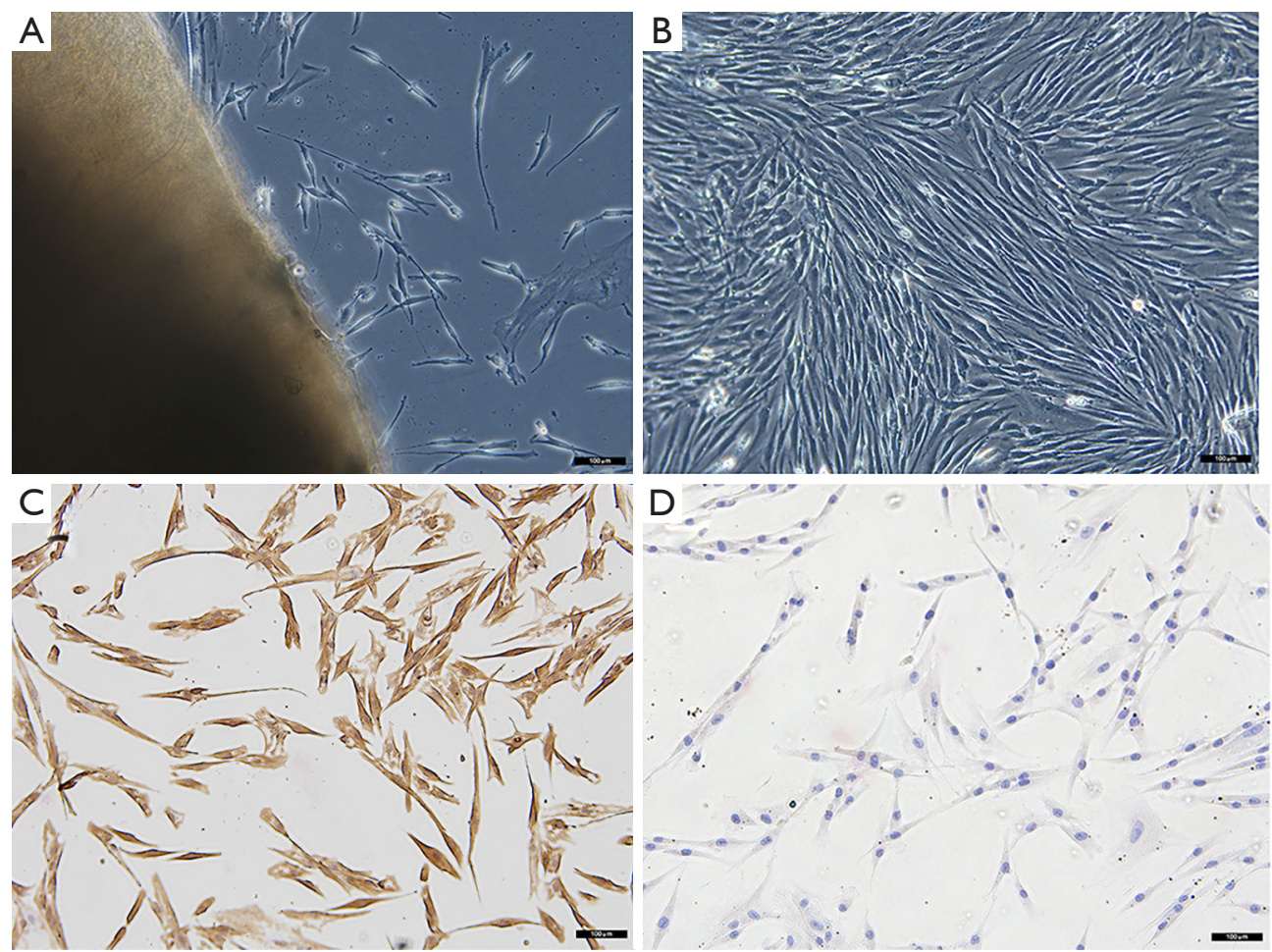

Figure 5 hGFCs culture and identification. (A) Primary hGFCs cultured in vitro, scale bar $100 \mu \mathrm{m}$. (B) $2^{\text {nd }}$ passage of the hGFCs, scale bar $100 \mu \mathrm{m}$. (C) hGFCs positive for vimentin antibody, scale bar $100 \mu \mathrm{m}$. (D) hGFCs negative for cytokeratin antibody, scale bar $100 \mu \mathrm{m}$. 
A

CCK-8
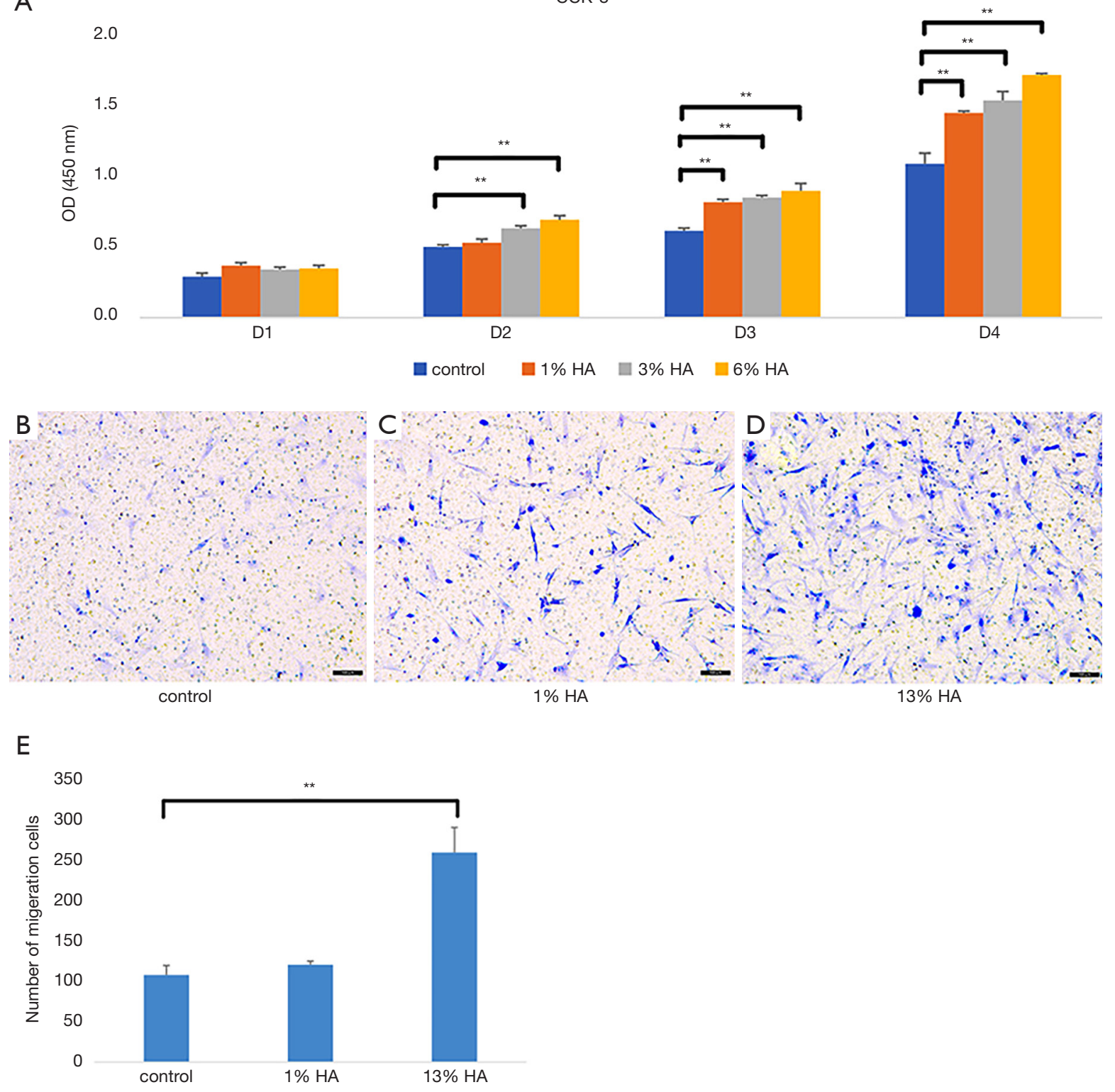

Figure 6 The proliferation and migration of hGFCs after HA treatment. (A) hGFCs were stimulated with $0 \%, 1 \%, 3 \%$ and $6 \%$ HA for 1, 2, 3 and 4 days. Asterisks indicate significant differences between the control group and HA stimulated group at different time points, ${ }^{* *} \mathrm{P}<0.001$. (B,C,D) Migration of hGFCs toward $0 \%, 1 \%$ and $13 \%$ HA, respectively, was evaluated with Transwell migration assay. The cells were stained with $0.1 \%$ crystal violet. Representative images are shown, scale bar $100 \mu \mathrm{m}$. (E) Cell migration was qualified by counting the cells on the lower side of the filter in three visual fields. Values are the mean $\pm \mathrm{SD}(\mathrm{n}=3),{ }^{* *} \mathrm{P}<0.001$. HA, hyaluronic acid.

group (Figure 6).

\section{Discussion}

HA, also called hyaluronan, is a major natural carbohydrate component of the extracellular matrix and is distributed widely in the joints, skin, eyes and connective tissues. The products currently in use and on the market are usually extracted from animal tissues such as rooster combs or obtained from certain bacterial strains (19). Meanwhile, the extracted HA must be chemically modified, such as via a cross-linking process, which could prevent quick 
degradation and prolong the effective time. The HA used in our study is produced from microbial fermentation.

Recently, many studies have reported that local application of HA adds to the benefits of nonsurgical and surgical periodontal therapy because of its antiinflammatory, antiedematous and antibacterial effects (20). HA is also labeled a dermal filler by plastic surgeons because of its unique ability to bind and retain water molecules (21). Hence, it has also been introduced to the field of esthetic dentistry to augment the gingival papilla and improve the esthetic aspects of the black triangles between teeth (9). Nevertheless, most of the studies on this topic have been case series or have lacked a control group, and only two RCTs with a parallel group design have been conducted. The results of these two studies are contradictory. Abdelraouf et al. demonstrated a significantly greater mean increase from baseline papilla height and decrease from baseline black triangle area in the HA group than in the control group after 3 and 6 months (22). However, the author did not clearly define tooth morphology or gingival biotype in the inclusion criteria, which are two important factors affecting the effects of gingival papilla treatment. Bertl et al. revealed that there was no clinically conspicuous increase in the volume of deficient papillae alongside implant-supported crowns in the anterior maxilla 6 months after the injection of HA (12). The different inclusion criteria, injection time points and HA products may account for the heterogeneity between the two studies.

In our previous study (14), we verified the remarkable effectiveness of HA gel (Qi Sheng Biological Agent Company Limited, Shanghai, China) injections in restoring the deficient gingival papillae of natural teeth, especially in patients with thick gingival biotypes. Nevertheless, that study had several shortcomings, such as a small sample size and a lack of a control group. Our present study sought to further evaluate the clinical results of HA gel injections versus saline injections in the treatment of gingival papilla defects with parallel in vivo and in vitro experiments. This is the first study with a split-mouth design in the literature on this topic; this design could reduce the impact of interindividual variability in patient characteristics that may impact the effects of treatment, such as age (23), crestal alveolar bone height (24), contact point (25), tooth shape (24), and interradicular distance (26). The results indicated that the HA gel group had improved significantly at 6 and 12 months relative to the baseline. This is also the first study to demonstrate the clinical efficacy of HA from both clinical and basic research perspectives. The two results support each other and illustrate that HA can effectively accelerate the proliferation and migration of gingival fibroblasts, ultimately increasing the height of deficient gingival papillae.

The improvement in deficient gingival papillae 12 months after saline injection is surprising. The underlying mechanism may be related to the natural creeping of gingival papilla and soft tissue regeneration caused by local tissue pressure. Previously, some studies reported that the application of compressive or retractive force could induce enlargement of the interdental papillae, especially at the site of closure of the extraction space. Nan et al. also demonstrated that mechanical force could promote the proliferation and extracellular matrix synthesis of human gingival fibroblasts (27). We assume that the injection of HA or saline solution applies some compressive force to gingival fibroblasts, which may also accelerate the proliferation of the cells and enhance the formation of ECM, improving gingival papilla defects.

This study has some limitations, such as the lack of information regarding patient satisfaction with and perception of the procedure, the lack of three-dimensional measurements of the gingival papillae, which would have improved the precision with which we could monitor the changes in black triangle size, and the overwhelming predominance of female patients, which prevented us from considering the influence of sex hormone levels on gingival papilla height and the inclusion of only maxillary sites. Additionally, the sample size is the most crucial issue in clinic trials, and we lacked a specific priori calculation process for sample size determination in the present study. However, we conducted back testing to evaluate the statistical power based on the data from this study. For the intragroup comparison, the statistical power obtained based on repeated measures was $98 \%$, indicating that there was a $98 \%$ chance of correctly rejecting the null hypothesis of no difference between the follow-up time points and baseline with a total of 60 gingival papilla defects. However, for the overall comparison between the HA gel intervention and the physiological saline solution control, the statistical power the statistical power obtained based on repeated measures was $64 \%$, indicating that there was a $36 \%$ chance of falsely retaining the null hypothesis of no difference with a total of 60 gingival papilla defects. Therefore, the result that there is no statistically significant additional benefit in the improvement of gingival papilla defects after HA injection compared with physiological saline injection must be interpreted with caution, and a larger sample size study is needed in the future. 


\section{Conclusions}

The present study confirms that the injection of HA could increase the gingival papilla height for gingival papilla defects and reduce the area of the resulting black triangles. However, there is no statistically significant additional benefit in the improvement of gingival papilla defects after HA injection comparing with physiological saline injection. In vitro study, the same HA product significantly accelerated the proliferation and migration of gingival fibroblasts. In the future, a long-term multicenter clinical study should be carried out to standardize the protocol for HA injection, optimize the injection concentration and interval, and identify the determinants affecting the clinical outcomes.

\section{Acknowledgments}

Funding: This work was supported by the Clinical Research Program of the $9^{\text {th }}$ People's Hospital affiliated with Shanghai Jiao Tong University School of Medicine (JYLJ201908) and by the Fundamental Research Program of the Ninth People's Hospital affiliated with Shanghai Jiao Tong University School of Medicine (JYZZ066). The funder is Shanghai Ninth People's Hospital affiliated with Shanghai Jiao Tong University School of Medicine.

\section{Footnote}

Reporting Checklist: The authors have completed the CONSORT reporting checklist. Available at http://dx.doi. org/10.21037/atm-20-7599

Data Sharing Statement: Available at http://dx.doi. org/10.21037/atm-20-7599

Conflicts of Interest: All authors have completed the ICMJE uniform disclosure form (available at http://dx.doi. org/10.21037/atm-20-7599). The authors have no conflicts of interest to declare.

Ethical Statement: The authors are accountable for all aspects of the work in ensuring that questions related to the accuracy or integrity of any part of the work are appropriately investigated and resolved. The study was conducted in accordance with the Declaration of Helsinki (as revised in 2013). The study was approved by the Shanghai Ninth People's Hospital Human Studies Ethics Committee
(No. 2016-229-T178). All participants were informed of the objective and risks of the study. Candidates were required to provide signed consent to participate.

Open Access Statement: This is an Open Access article distributed in accordance with the Creative Commons Attribution-NonCommercial-NoDerivs 4.0 International License (CC BY-NC-ND 4.0), which permits the noncommercial replication and distribution of the article with the strict proviso that no changes or edits are made and the original work is properly cited (including links to both the formal publication through the relevant DOI and the license). See: https://creativecommons.org/licenses/by-nc-nd/4.0/.

\section{References}

1. Bichacho N. Papilla regeneration by noninvasive prosthodontic treatment: segmental proximal restorations. Pract Periodontics Aesthet Dent 1998;10:75, 77-8.

2. Cardaropoli D, Re S, Corrente G. The Papilla Presence Index (PPI): a new system to assess interproximal papillary levels. Int J Periodontics Restorative Dent 2004;24:488-92.

3. Carnio J, Carnio AT. Papilla reconstruction: Interdisciplinary consideration for clinical success. J Esthet Restor Dent 2018;30:484-91.

4. Ahila E, Saravana Kumar R, Reddy VK, et al. Augmentation of Interdental Papilla with Platelet-rich Fibrin. Contemp Clin Dent 2018;9:213-7.

5. Muthukumar S, Rangarao S. Surgical augmentation of interdental papilla - A case series. Contemp Clin Dent 2015;6:S294-8.

6. Feuillet D, Keller JF, Agossa K. Interproximal Tunneling with a Customized Connective Tissue Graft: A Microsurgical Technique for Interdental Papilla Reconstruction. Int J Periodontics Restorative Dent 2018;38:833-9.

7. Henriques PG, Okajima LS, Siqueira S Jr. Surgical reconstruction of the interdental papilla: 2 case reports. Gen Dent 2018;66:e1-4.

8. Yadav D, Singh S, Roy S. Periodontal microsurgery for management of multiple marginal tissue recession using Zucchelli's modification of coronally advanced flap and pericardium membrane in an esthetic zone. J Indian Soc Periodontol 2019;23:284-9.

9. Becker W, Gabitov I, Stepanov M, et al. Minimally invasive treatment for papillae deficiencies in the esthetic zone: a pilot study. Clin Implant Dent Relat Res 2010;12:1-8. 
10. Pi S, Choi YJ, Hwang S, et al. Local Injection of Hyaluronic Acid Filler Improves Open Gingival Embrasure: Validation Through a Rat Model. J Periodontol 2017;88:1221-30.

11. Awartani FA, Tatakis DN. Interdental papilla loss: treatment by hyaluronic acid gel injection: a case series. Clin Oral Investig 2016;20:1775-80.

12. Bertl K, Gotfredsen K, Jensen SS, et al. Can hyaluronan injections augment deficient papillae at implant-supported crowns in the anterior maxilla? A randomized controlled clinical trial with 6 months follow-up. Clin Oral Implants Res 2017;28:1054-61.

13. Asparuhova MB, Kiryak D, Eliezer M, et al. Activity of two hyaluronan preparations on primary human oral fibroblasts. J Periodontal Res 2019;54:33-45.

14. Ni J, Shu R, Li C. Efficacy Evaluation of Hyaluronic Acid Gel for the Restoration of Gingival Interdental Papilla Defects. J Oral Maxillofac Surg 2019;77:2467-74.

15. Saito A, Bizenjima T, Takeuchi T, et al. Treatment of intrabony periodontal defects using rhFGF-2 in combination with deproteinized bovine bone mineral or rhFGF-2 alone: A 6-month randomized controlled trial. J Clin Periodontol 2019;46:332-41.

16. Nordland WP, Tarnow DP. A classification system for loss of papillary height. J Periodontol 1998;69:1124-6.

17. Chapple ILC, Mealey BL, Van Dyke TE, et al. Periodontal health and gingival diseases and conditions on an intact and a reduced periodontium: Consensus report of workgroup 1 of the 2017 World Workshop on the Classification of Periodontal and Peri-Implant Diseases and Conditions. J Periodontol 2018;89 Suppl 1:S74-84.

18. Moffitt ML, Bencivenni D, Cohen RE. Drug-induced gingival enlargement: an overview. Compend Contin Educ Dent 2013;34:330-6.

Cite this article as: $\mathrm{Ni}$ J, Zhong Z, Wu Y, Shu R, Wu Y, Li C. Hyaluronic acid vs. physiological saline for enlarging deficient gingival papillae: a randomized controlled clinical trial and an in vitro study. Ann Transl Med 2021;9(9):759. doi: 10.21037/atm20-7599
19. Al-Khateeb R, Olszewska-Czyz I. Biological molecules in dental applications: hyaluronic acid as a companion biomaterial for diverse dental applications. Heliyon 2020;6:e03722.

20. Eliezer M, Imber JC, Sculean A, et al. Hyaluronic acid as adjunctive to non-surgical and surgical periodontal therapy: a systematic review and meta-analysis. Clin Oral Investig 2019;23:3423-35.

21. Fallacara A, Manfredini S, Durini E, et al. Hyaluronic Acid Fillers in Soft Tissue Regeneration. Facial Plast Surg 2017;33:87-96.

22. Abdelraouf SA, Dahab OA, Elbarbary A, et al. Assessment of Hyaluronic Acid Gel Injection in the Reconstruction of Interdental Papilla: A Randomized Clinical Trial. Open Access Maced J Med Sci 2019;7:1834-40.

23. Chang LC. The association between embrasure morphology and central papilla recession. J Clin Periodontol 2007;34:432-6.

24. Chen MC, Liao YF, Chan CP, et al. Factors influencing the presence of interproximal dental papillae between maxillary anterior teeth. J Periodontol 2010;81:318-24.

25. Tarnow DP, Magner AW, Fletcher P. The effect of the distance from the contact point to the crest of bone on the presence or absence of the interproximal dental papilla. J Periodontol 1992;63:995-6.

26. Martegani P, Silvestri M, Mascarello F, et al. Morphometric study of the interproximal unit in the esthetic region to correlate anatomic variables affecting the aspect of soft tissue embrasure space. J Periodontol 2007;78:2260-5.

27. Nan L, Zheng Y, Liao N, et al. Mechanical force promotes the proliferation and extracellular matrix synthesis of human gingival fibroblasts cultured on 3D PLGA scaffolds via TGFbeta expression. Mol Med Rep 2019;19:2107-14. 